Check for updates

Cite this: RSC Adv., 2020, 10, 23668

\title{
Automated glycan assembly of Streptococcus pneumoniae type 14 capsular polysaccharide fragments $\dagger$
}

\begin{abstract}
João Louçano, ${ }^{a}$ Peter Both, ${ }^{\mathrm{b}}$ Andrea Marchesi, (iD ${ }^{\mathrm{b}}$ Linda del Bino, ${ }^{\mathrm{c}}$ Roberto Adamo, (iD c Sabine Flitsch (iD ${ }^{b}$ and Mario Salwiczek (iD *a

S. pneumoniae is a major human pathogen with increasing antibiotic resistance. Pneumococcal vaccines consist of capsular polysaccharide (CPS) or their related fragments conjugated to a carrier protein. The repeating unit of $S$. pneumoniae type 14 CPS shares a core structure with the CPS of Group B Streptococcus (GBS) type III: the only difference is that the latter exhibits a sialic acid unit, with a $\alpha-2,3$ linkage to galactose. Here, the automated glycan assembly (AGA) of two frameshifts of the repeating unit of S. pneumoniae type 14 is described. The same strategy is used to assemble dimers of the different repeating unit frameshifts. The four structures are assembled with only three commercially available monosaccharide building blocks. We also report an example of how enzymatic sialylation of the compounds obtained with AGA completes a synthetic route for GBS type III glycans. The synthesized structures were tested in competitive ELISA and further confirmed the branched tetrasaccharide GalGlc-(Gal-)GlcNAc to be the minimal epitope of S. pneumoniae type 14.
\end{abstract}

Received 25th February 2020

Accepted 13th June 2020

DOI: 10.1039/d0ra01803a

rsc.li/rsc-advances buildings blocks, optimizing a synthetic procedure can now be achieved within much smaller time frames. Given the demand for structurally defined synthetic carbohydrates in glycobiology and immunology, we engaged on an optimization study for the synthesis of $S$. pneumoniae capsular polysaccharide.

$S$. pneumoniae is a leading cause of serious invasive diseases such as bacterial pneumonia, septicaemia, and meningitis in

carbohydrate polymers prepared by AGA has been increasing continuously and structurally diverse antigens, including the Lewis antigens, ${ }^{3}$ oligosaccharides containing multiple cis-linkages, ${ }^{4}$ glycosaminoglycans, ${ }^{5}$ and long oligosaccharides (e.g. a mannose $50-$ mer $^{6}$ and $150-$ mer $^{7}$ ) have been reported. Success in the overall AGA process requires an optimized and highly efficient chemistry. The main challenge is to achieve highly efficient individual couplings to yield a specific stereochemistry for each of the emerging glycosidic linkages. The vast structural diversity of the monomeric building blocks, however, implicates equally diverse physicochemical properties and as such, the development of a reproducible synthetic protocol often requires a time-consuming iterative approach to optimize each coupling step. With the development of AGA and an increasing number of commercially available ready-to-use monomeric

${ }^{a}$ GlycoUniverse GmbH \& Co KGaA, Am Mühlenberg 11, 14476 Potsdam, Germany. E-mail:m.salwiczek@glycouniverse.de

${ }^{b}$ School of Chemistry, University of Manchester, Manchester Institute of Biotechnology, 131 Princess Street, Manchester M1 7DN, UK

${ }^{c}$ GSK, Via Fiorentina 1, 53100 Siena, Italy

$\dagger$ Electronic supplementary information (ESI) available. See DOI: 10.1039/d0ra01803a

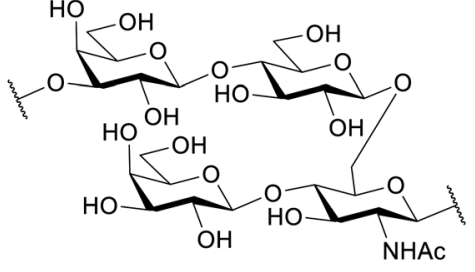

S. pneumoniae type 14 CPS repeating unit

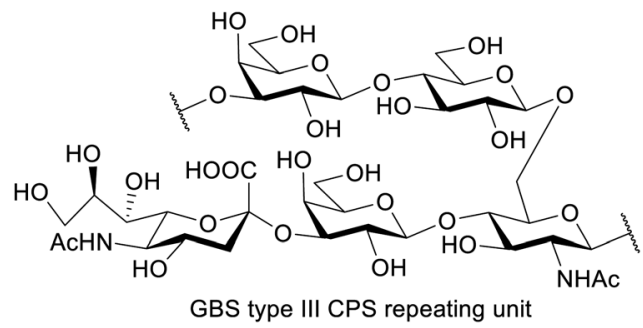

Fig. 1 Repeating unit of the capsular polysaccharide of S. pneumoniae type 14 and GBS type III. 
young children worldwide, and is listed in the WHO global priority list of antibiotic-resistant bacteria. ${ }^{8}$ Rising levels of antibiotic resistance reinforce the need for alternative treatments and prevention strategies such as vaccines. The capsular polysaccharide (CPS) is a major virulence factor and the basis of pneumococcal vaccines. Pneumococcal conjugate vaccines contain ten or thirteen CPS types, including type $14 .^{9}$ The repeating unit of $S$. pneumoniae type 14 CPS (Sp14 CPS $)^{\mathbf{1 0}}$ is depicted in Fig. 1. This tetrasaccharide is the minimal immunogenic polysaccharide portion that elicits a protective response. ${ }^{\mathbf{1 1 2} 12}$ A conjugate of this minimal epitope showed strong immunogenicity in mice, also as multivalent vaccine formulation combined with conjugates from other synthetic pneumococcal carbohydrate antigens. ${ }^{13}$

Interestingly, the repeating unit of Sp14 CPS shares high structural identity with that of Group B Streptococcus (GBS) type III capsule, which exhibits an additional Neu5Ac residue $\alpha(2-3)$ linked to the upstream Gal residue. ${ }^{14}$ GBS is a leading cause of sepsis and meningitis in newborns and causes an estimated 147000 annual stillbirths and infant deaths globally. ${ }^{15}$ GBS capsule is a virulence factor and an important target for vaccine development. ${ }^{16}$ Ten different serotypes differentiated based on their structurally distinct sialylated polysaccharide capsules are known (Ia, Ib, II to IX) and all are associated with human disease. Serotype III is the most prevalent causing more than half of the infection cases. ${ }^{17}$

Pneumococcal antigens have been previously synthesized by solution-phase classical methods ${ }^{\mathbf{1 8 , 1 9}}$ and AGA. ${ }^{20}$ One solution phase strategy to prepare Sp14 used a thioglycoside tetrasaccharide block, corresponding to the repeating unit of Sp14. The coupling of this block with a spacer, followed by removal of an isopropylidene acetal, yielded an acceptor that was elongated with the donor block to give a protected dimer of the repeating unit. $^{21}$ Other strategies include the use of differentially protected lactose and lactosamine building blocks that were coupled to provide fragments up to a length of eight monosaccharides. ${ }^{22}$ For the synthesis of GBS structures that contain a terminal sialic acid, enzymatic methods offer an effective alternative to the usually low yielding chemical methods. ${ }^{23}$ The synthesis of the GBSIII repeating unit has been achieved by enzymatic introduction of the sialic acid in Sp14 structures. ${ }^{24,25}$ To avoid sialylation in the lactose arm of the structure, the corresponding galactose was blocked with a methyl ether in position 3 that could, however, not be removed in the end of the synthesis. Boons et al. described a fully chemical synthesis of a heptasaccharide fragment of GBS type III based on
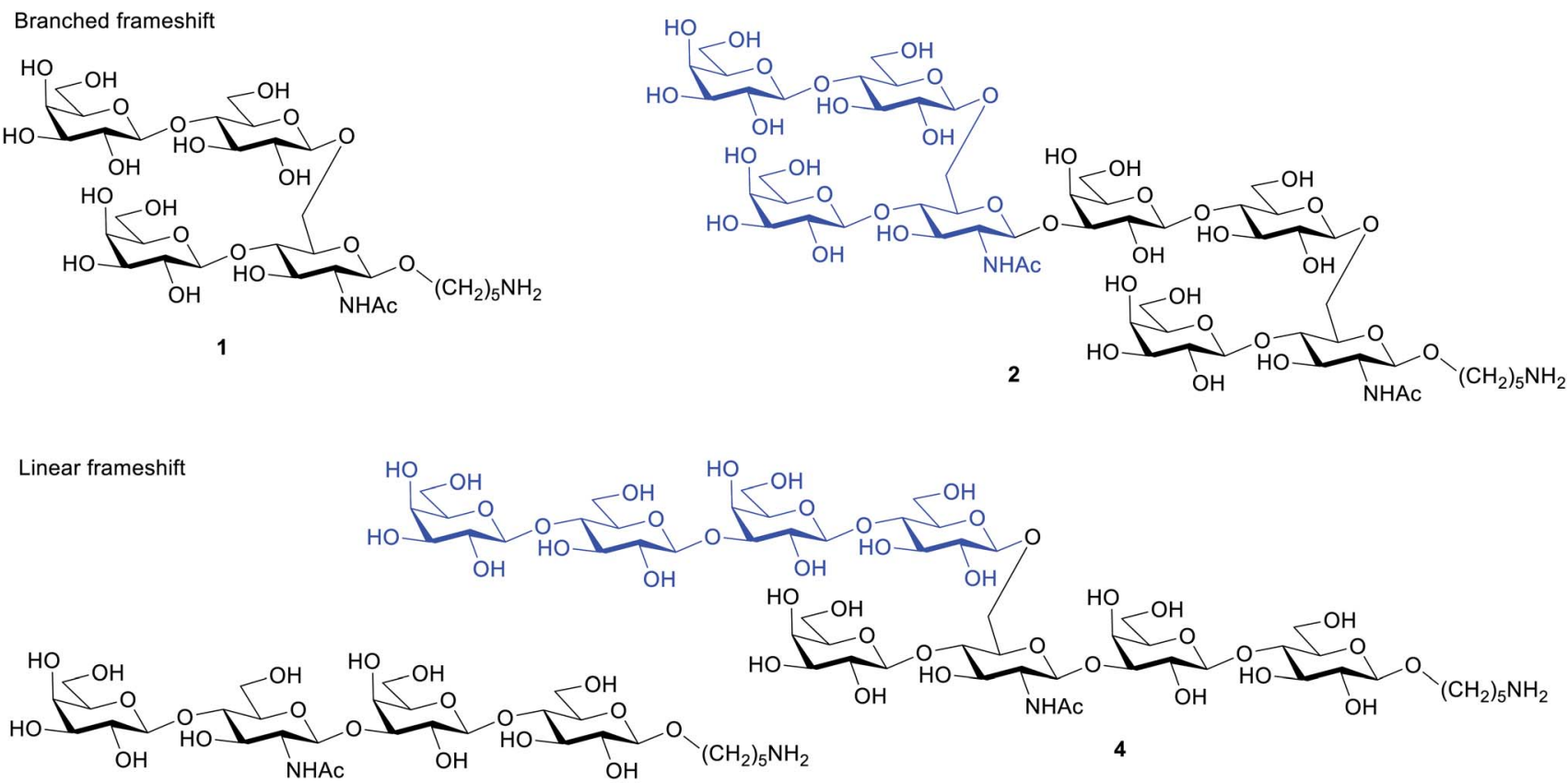

3
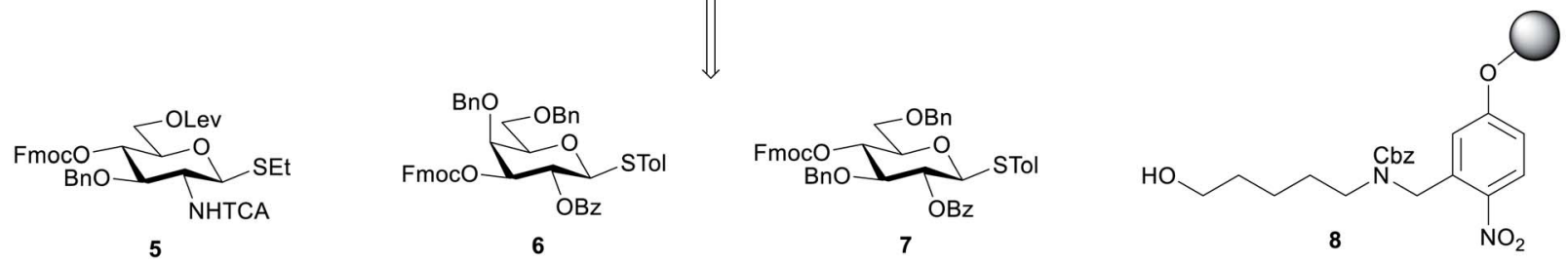

Scheme 1 Retrosynthesis of oligosaccharides 1-4 using building blocks 5-7 and the functionalized Merrifield resin 8. 
a convergent approach. The incorporation of sialic acid was based on $\alpha$-NeuNAc-(2-3)-Galp methylthioglycoside disaccharide which had been assembled by coupling a methylthiosialoside and a galactose acceptor. Similarly, a series of GBSIII fragments have been recently assembled chemically to map the sialylated GBSIII structural epitope. ${ }^{26,27}$

Here, we take the repeating unit of Sp14 as a target structure and describe the optimization of AGA for this bacterial glycan. We proceed to show an example of enzymatic sialylation of one of the obtained structures to generate the GBS type III CPS repeating unit.

\section{Results and discussion}

\section{AGA}

The target structures for AGA (Scheme 1) represent two different frameshifts of the repeating unit (monomer) and the corresponding dimer of Sp14. In the branched frameshift (compounds 1 and 2), the reducing terminus is comprised of glucosamine. In the linear frameshift (compounds 3 and $\mathbf{4}$ ), glucose lies at the reducing terminus. Our retrosynthetic analysis of the target structures led to three thioglycoside building blocks that can be used to make both frameshifts. In our strategy, Fmoc served as the main temporary protecting group whereas Lev was used as an orthogonal temporary protecting to enable branching (glucosamine). Benzyl ether groups were used as permanent protecting groups. Benzoyl ester, and for the aminosugar, trichloroacetyl amide (NHTCA) at C2 were used as participating protecting groups to ensure selective trans-glycosylation. One galactose building block (6) was used both for terminal positions and where $\mathrm{O} 3$ elongation was required. In the first case, a capping step was used right after deprotecting the Fmoc group giving rise to a 3-O-acetylated galactose residue. This strategy allows time saving during the set-up of the synthesis and proves the versatility of the building block.

The elongation cycle to incorporate each building block on the Glyconeer is comprised of four pre-programmed modules: acid wash, glycosylation, capping and deprotection (Table 1). The capping procedure prevents the growth of deletion sequences which facilitates purification and increases yield of the target product. ${ }^{28}$ Fmoc and Lev groups were cleaved as previously reported. ${ }^{28}$ The success of AGA depends largely on very high individual coupling yields which can be achieved by optimizing the key parameters related to the glycosylation module: temperature of glycosylation, number of coupling repetitions, and excess of donor.

We defined the initial standard conditions as: $12.5 \mu \mathrm{mol}$ scale (based on linker loading); glycosylations using $2 \times 6.5$ equivalents ( $41 \mathrm{mM}$ ) of donor, $T_{1}=-20{ }^{\circ} \mathrm{C}$ and $T_{2}=0{ }^{\circ} \mathrm{C}$. After AGA and photocleavage to release the glycans from the solid support, the crude material was analysed by HPLC evaluate the success of the synthesis and the target product was purified by preparative HPLC. With these conditions, the target tetrasaccharide 9 was obtained in $12 \%$ yield (Table 2, entry 1 ). The HPLC analysis of the crude product revealed a significant contamination with the capped linker (see ESI $\dagger$ ). When the first building block 5 was coupled using ten equivalents (62 mM), the signal for the capped linker was negligible and tetrasaccharide 9 was isolated in $20 \%$ yield (Table 2, entry 2). This finding suggests that the glucosamine building block 5 requires a more vigorous approach to achieve good coupling yield. Before proceeding to the assemble of the dimer 10, the synthesis of oligosaccharide $\mathbf{9}$ was repeated using ten equivalents of donor in one single coupling for building blocks 6 and 7. The crude material obtained after photocleavage showed negligible deletion sequences (see HPLC in ESI $\dagger$ ) and the

Table 2 Conditions for AGA of tetrasaccharide 9

\begin{tabular}{llllll}
\hline Entry & BB & equiv. & $T_{1}$ & $T_{2}$ & Isolated yield \\
\hline 1 & $\mathbf{5}$ & $2 \times 6.5$ & -20 & 0 & $12 \%$ \\
& $\mathbf{6}$ & $2 \times 6.5$ & -20 & 0 & \\
& 7 & $2 \times 6.5$ & -20 & 0 & \\
& $\mathbf{6}$ & $2 \times 6.5$ & -20 & 0 & \\
& $\mathbf{5}$ & $2 \times 10$ & -20 & 0 & $20 \%$ \\
& $\mathbf{6}$ & $2 \times 6.5$ & -20 & 0 & \\
& 7 & $2 \times 6.5$ & -20 & 0 & \\
& $\mathbf{6}$ & $2 \times 6.5$ & -20 & 0 & \\
& $\mathbf{5}$ & $2 \times 10$ & -20 & 0 & $36 \%$ \\
& $\mathbf{6}$ & $1 \times 10$ & -20 & 0 & \\
& 7 & $1 \times 10$ & -20 & 0 & \\
& $\mathbf{6}$ & $1 \times 10$ & -20 & 0 &
\end{tabular}

Table 1 Pre-programmed modules used in the Glyconeer for each elongation cycle

Module

(1) Acid Wash

(2) Glycosylation

(3) Capping

(4) Deprotection
Description

Resin is washed with a solution of TMSOTf in DCM Building block and the activator solution (NIS/TfOH) are delivered to the reaction vessel at temperature $T_{1}$ and incubated for $5 \mathrm{~min}$. The temperature is then raised to $T_{2}$ and an incubation time of $20 \mathrm{~min}$ is followed

Resin is capped by acetylation with a solution of acetic anhydride and methanesulfonic acid in DCM preceded by a short wash with $10 \%$ pyridine in DMF

Temporary protecting group is deprotected. Fmoc is cleaved with a solution of $20 \%(\mathrm{v} / \mathrm{v})$ piperidine in DMF for $5 \mathrm{~min}$. Lev is deprotected with a $0.15 \mathrm{M}$ solution of hydrazine acetate in $\mathrm{Py} / \mathrm{AcOH} / \mathrm{H}_{2} \mathrm{O}(3 \times 30 \mathrm{~min})$ 
Table 3 Conditions for AGA of octasaccharide 10

\begin{tabular}{llllll}
\hline & & & & & \\
Entry & BB & equiv. & $T_{1}$ & $T_{2}$ & $\begin{array}{l}\text { Isolated } \\
\text { yield }\end{array}$ \\
\hline 1 & $\mathbf{5}$ & $2 \times 10$ & -20 & 0 & $1 \%$ \\
& $\mathbf{6}$ & $1 \times 10$ & -20 & 0 & \\
& 7 & $1 \times 10$ & -20 & 0 & \\
& $\mathbf{6}$ & $1 \times 10$ & -20 & 0 & \\
& $(2 \times)$ & & & & \\
2 & $\mathbf{5}$ & $2 \times 10$ & $(\mathrm{i})-20$ (ii) -10 & (i) 0 & $4 \%$ \\
& & & & $($ ii) 10 & \\
& $\mathbf{6}$ & $1 \times 6.5$ & -20 & 0 & \\
& 7 & $2 \times 6.5$ & -20 & 0 & \\
& $\mathbf{6}$ & $1 \times 6.5$ & -20 & 0 & \\
& $(2 \times)$ & & & & \\
3 & $\mathbf{5}$ & $(\mathrm{i}) 2 \times(\mathrm{ii}) 4 \times 10$ & $(\mathrm{i})-20$ (ii) 0 & $(\mathrm{i}) 0(\mathrm{ii}) 20$ & $8 \%$ \\
& $\mathbf{6}$ & $1 \times 6.5$ & -20 & 0 & \\
& 7 & $2 \times 6.5$ & -20 & 0 & \\
& $\mathbf{6}$ & $1 \times 6.5$ & -20 & 0 & \\
& $(2 \times)$ & & & &
\end{tabular}

product was isolated in $31 \%$ yield (Table 2, entry 3 ). With these conditions, the synthesis time was reduced from 13.5 to $10.5 \mathrm{~h}$ and 2.5 equivalents per coupling of building block 6 and 7 were saved.

These conditions were then applied for the synthesis of the dimer 10 (Table 3, entry 1). Unfortunately, the major product was the deletion sequence tetrasaccharide 14 (Fig. 2). The formation of 14 can be explained by a very low coupling efficiency of glucosamine building block 5 to the $\mathrm{O} 3$ position of galactose. In an attempt to improve this step, the effect of the glycosylation temperature for building block $\mathbf{5}$ was probed: the temperatures $T_{1}=-10{ }^{\circ} \mathrm{C}$ and $T_{2}=0{ }^{\circ} \mathrm{C}$ led to an increase in the formation of the target octasaccharide; $T_{1}=0{ }^{\circ} \mathrm{C}$ and $T_{2}=20{ }^{\circ} \mathrm{C}$ led to a decrease in the formation of the target octasaccharide. However, even with the best temperature set (Table 3, entry 2) the deletion sequence $\mathbf{1 4}$ prevailed as the major product. When glucosamine 5 was coupled at $-10{ }^{\circ} \mathrm{C}$ to $10{ }^{\circ} \mathrm{C}$ and repeated in
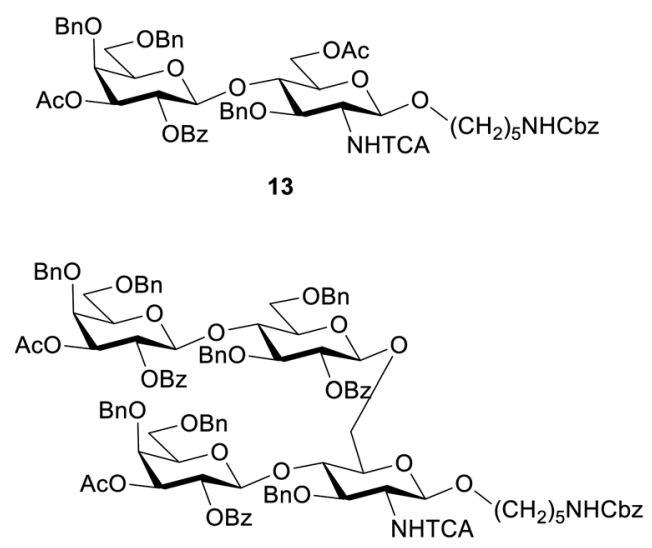

14

Fig. 2 Deletion sequences identified in the synthesis of octasaccharide 10 . a quadruple coupling, the synthesis proceeded with the formation of $\mathbf{1 0}$ as the major product (Table 3, entry 3, 8\% yield). Building block 7 was used again in a double coupling to prevent the formation of deletion sequence $\mathbf{1 3}$ and the amount of building block 6 was reduced to 6.5 equiv. without formation of deletion sequences.

To generate the fragments of the linear frameshift, the best conditions for the synthesis of dimer $\mathbf{1 0}$ were used. The synthesis of tetrasaccharide 11 (Table 4, entry 1 ) was successful and led to $23 \%$ yield. The synthesis of 12 was performed by repeating the elongation cycles. An unexpected deletion sequence was identified as a tetrasaccharide with the Lev still present at the branching point at $\mathrm{C} 6$ of glucosamine indicating incomplete cleavage of this protecting group. Nevertheless, the octasaccharide could still be isolated in $8 \%$ yield (Scheme 2).

\section{Upscaling on the Glyconeer}

The AGA synthesis described so far refer to a $12.5 \mu \mathrm{mol}$ scale. A linear scale-up to $25 \mu \mathrm{mol}$ was performed with the conditions in Table 2, entry 2 and the target tetrasaccharide 9 was isolated in $28 \%$. The synthesis of the tetrasaccharide $\mathbf{1 1}$ was also linearly scaled up to $25 \mu \mathrm{mol}$ using the conditions in Table 4, entry 1 , leading to an isolated yield of $21 \%$. These are comparable to the lower scale experiments (20\% and $23 \%$, respectively). The synthesis of tetrasaccharide 9 was further called up to $50 \mu \mathrm{mol}$. In this case, due to volume limitations, the amount of BBs was reduced to $2 \times 5$ equivalents per coupling, keeping the concentration at $41 \mathrm{mM}$. Interestingly, this time, tetrasaccharide 9 was isolated in $33 \%$.

\section{Deprotection}

Following AGA, cleavage from the solid support, and HPLC purification, compounds 9, 10, 11 and 12 were deprotected in two steps. Ester groups (benzoyl, acetyl and levaloyl) were cleaved with sodium methoxide in 1:1 MeOH/DCM. In the second step, benzyl ethers, NHTCA and the carboxybenzyl group at the amino linker were removed by hydrogenation catalyzed by $\mathrm{Pd} / \mathrm{C}$. Methanolysis of tetrasaccharide 9 was complete after $24 \mathrm{~h}$ using $25 \mathrm{mM}$ NaOMe (by MALDI). The crude product was then hydrogenated and purified using a C18 cartridge to give 1 in $93 \%$ overall deprotection yield. The linear

Table 4 Conditions for AGA of tetrasaccharide 11 and octasaccharide 12

\begin{tabular}{llllll}
\hline Entry & BB & equiv. & $T_{1}$ & $T_{2}$ & Isolated yield \\
\hline 1 & 7 & $2 \times 6.5$ & -20 & 0 & $23 \%(\mathbf{1 1})$ \\
& $\mathbf{6}$ & $1 \times 6.5$ & -20 & 0 & \\
& $\mathbf{5}$ & $4 \times 10$ & -10 & 10 & \\
& $\mathbf{6}$ & $1 \times 6.5$ & -20 & 0 & \multirow{2}{*}{$8 \%(\mathbf{1 2})$} \\
& 7 & $2 \times 6.5$ & -20 & 0 & \\
& $\mathbf{6}$ & $1 \times 6.5$ & -20 & 0 & \\
& $\mathbf{5}$ & $4 \times 10$ & -10 & 10 &
\end{tabular}

$(2 \times)$ 


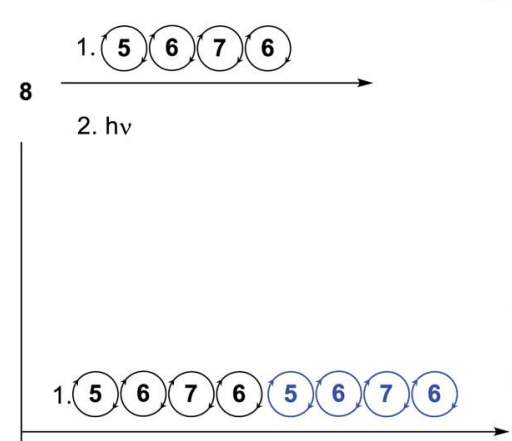

2. hv
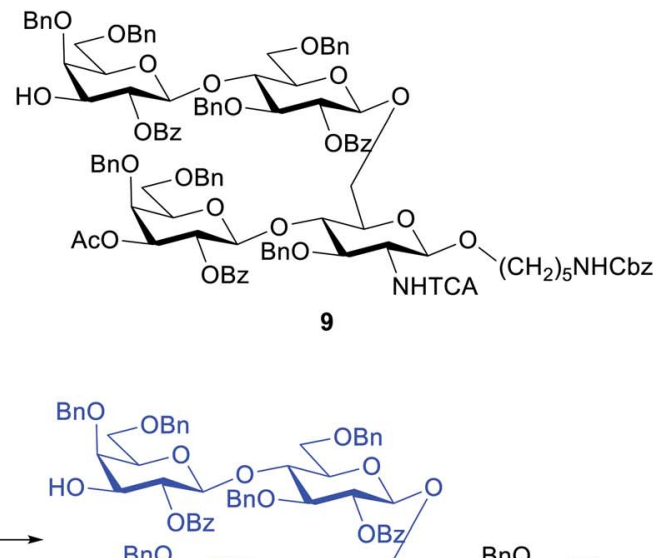

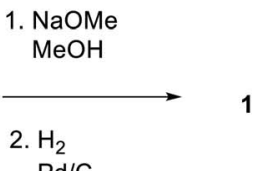

$\mathrm{Pd} / \mathrm{C}$

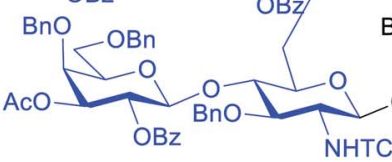

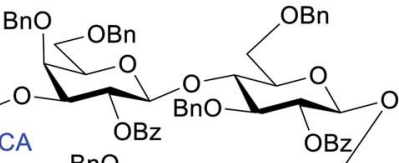

1. $\mathrm{NaOMe}$

$\mathrm{MeOH}$

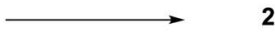

2. $\mathrm{H}_{2}$

$\mathrm{Pd} / \mathrm{C}$

10
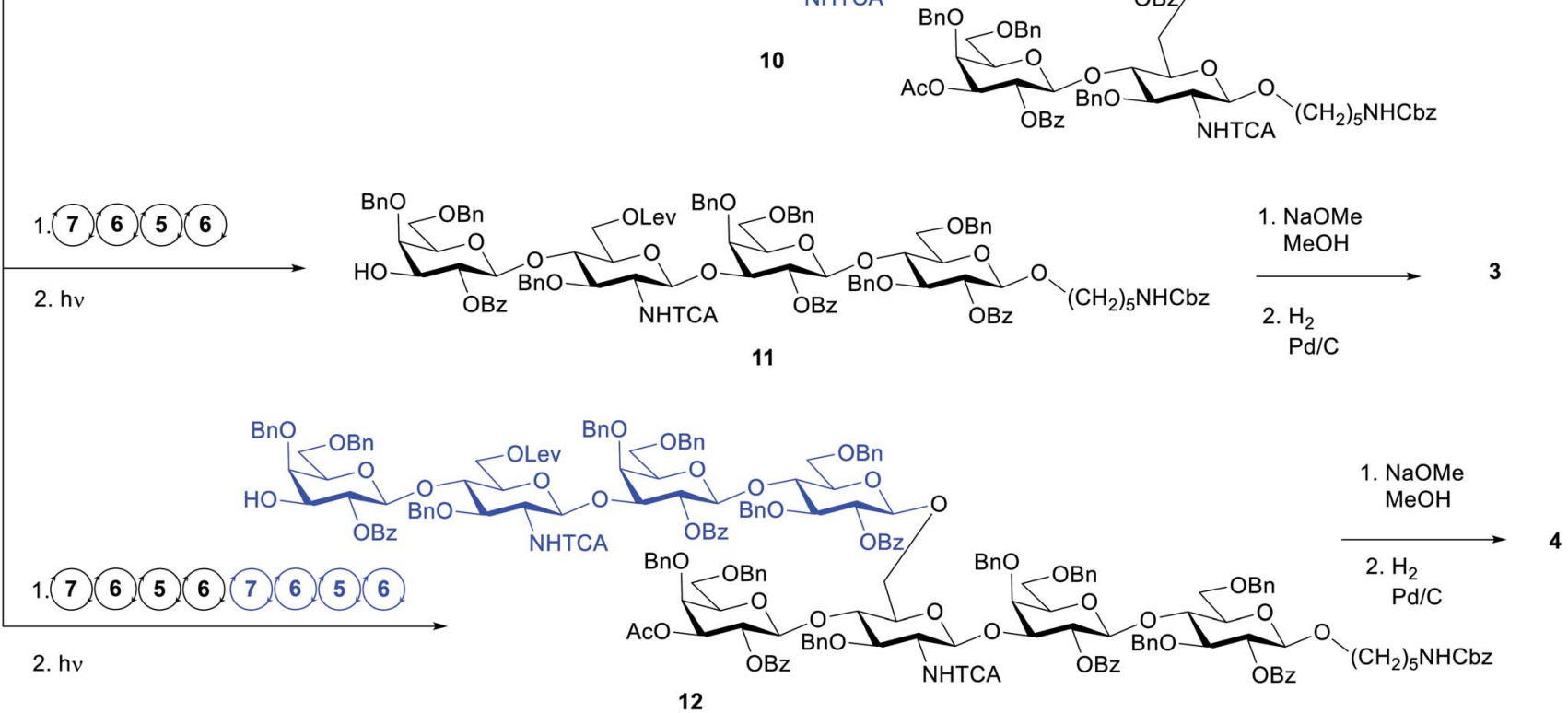

Scheme 2 AGA of oligosaccharides 9-12, and global deprotection to generate 1-4. Circular arrows represent the elongation cycle for each BB (Table 1).

tetrasaccharide $\mathbf{1 1}$ was submitted to the same methanolysis procedure. However, after $24 \mathrm{~h}$, mass analysis showed partially cleaved intermediates and when left to react for 4 days the sample degraded. When performed with an increased concentration of NaOMe (50 mM), the reaction showed similar results. Upon addition of another portion of NaOMe (final concentration $0.1 \mathrm{M}$ ) and after another $24 \mathrm{~h}$, the conversion was complete. The crude product was then subjected to hydrogenation. Purification using a C18 cartridge was not sufficient and tetrasaccharide 3 was obtained after HPLC purification as the formate salt ( $71 \%$ overall deprotection yield).

Deprotection of octasaccharides $\mathbf{1 0}$ and $\mathbf{1 2}$ proved to be cumbersome as the methanolysis could not be followed by LCMS or MALDI. For branched structure 10, the procedure used for methanolysis was the same as for the branched tetrasaccharide 9. After hydrogenation, the crude product was subjected to RP-HPLC but was isolated with a triethylamine contamination, originating from the work up procedure. The

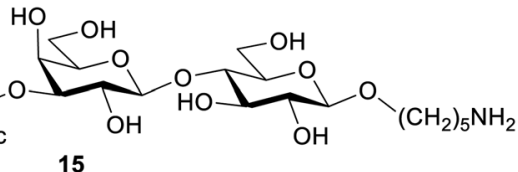

Scheme 3 Enzymatic synthesis of sialoside 14. $\alpha-(2,3)$-Sialyltransferase PmST1, CMP-Neu5Ac, alkaline phosphatase, Tris-HCl pH 9.3, MgCl 2. 
linear structure 12 was deprotected using the same conditions as for linear tetrasaccharide 11. After hydrogenation and RPHPLC purification, the product was isolated in $22 \%$ yield (overall deprotection yield).

\section{Enzymatic sialylation}

To convert the $S$. pneumoniae type 14 repeating unit (oligosaccharide 3) into the GBS type III repeating unit (compound 15), an $\alpha(2,3)$-sialyltransferase from Pasteurella multocida (PmST1) ${ }^{29}$ was used. The reaction was performed using three equivalents of CMP-Neu5Ac ( $3 \mathrm{mM}$ ) as donor in the presence of an alkaline phosphatase to dephosphorylate the CMP by-product. The enzyme was used in a concentration of $6 \mu \mathrm{g} \mathrm{mL} \mathrm{m}^{-1}$ and after incubation at $37^{\circ} \mathrm{C}$ with a Tris- $\mathrm{HCl}$ buffer $(\mathrm{pH} 9.3)$, the sialoside was filtered by a polyethersulfone membrane (MWCO $10 \mathrm{kDa}$ ) to remove the protein. A final purification by RP-HPLC gave 15 in $30 \%$ yield (Scheme 3 ).

\section{Antigenic evaluation of the synthesized pneumococcal glycans}

The antigenicity of the synthesized pneumococcal serotype 14 fragments was assessed by competitive ELISA (Fig. 3). To this end, plates were coated with Sp14 polysaccharide and binding to the corresponding murine anti serum was inhibited with serial dilutions of the synthesized oligosaccharides 1-3 and 15. The natural polysaccharide Sp14 was the positive control, while the GBSIII capsular polysaccharide was the negative control. The branched structure $\mathbf{1}$ and its dimer $\mathbf{2}$ were able to fully inhibit the binding of the Sp14 serum to the polysaccharide. In contrast, the linear glycan 3 and its sialylated form 15 resembling the GBSIII polysaccharide linear frameshift failed to inhibit the binding. These results further support the theory whereby the minimal immunogenic epitope of Sp14 is the branched tetrasaccharide $\mathbf{1}^{\mathbf{1 1}}$ The lack of specificity of the fragments 1 and 2 towards anti GBSIII antibodies was also confirmed by inhibiting the binding of a rabbit GBSIII mAb (Fig. S1, ESI $\dagger$ ), that has been shown to recognize a sialylated GBSIII portion comprised of two repeating units. ${ }^{27}$ While GBSIII polysaccharide at the tested dilutions showed to deplete the

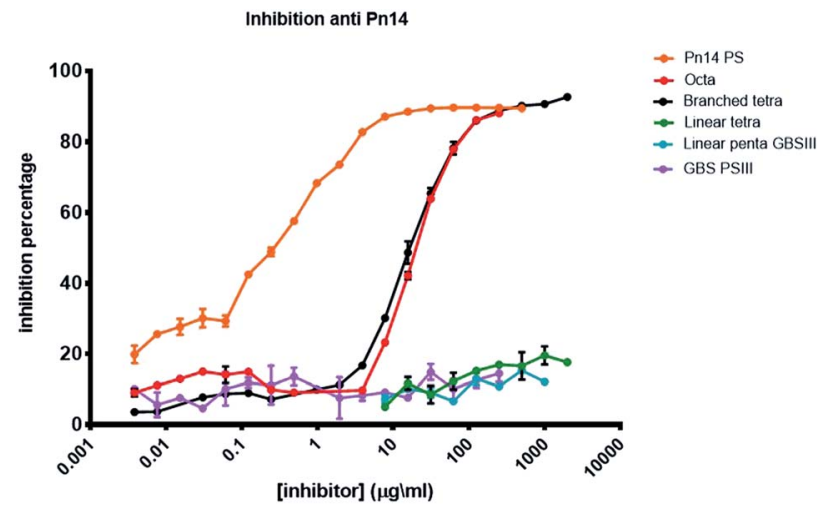

Fig. 3 Inhibition percentage of Sp14 capsular polysaccharide using a specific murin serum (saccharide concentration expressed as $\mu \mathrm{g}$ $\mathrm{mL}^{-1}$ ).
mAb recognition, the two pneumococcal fragments did not. This result agrees with the previous finding that anti-GBSIII human antibodies recognize a shared linear structure in the backbone of both Sp14 and GBSIII, represented by the -GlcGlcNAc-Gal-motif. ${ }^{30}$

\section{Conclusions}

The success of AGA relies on general and standardized procedures. The coupling of BBs 5-7 was explored with the best conditions leading to reproducible good coupling yields in two different frameshifts of Sp14 CPS oligosaccharides. This showcases the importance of coupling optimization and proves the versatility the monosaccharides building blocks, setting the scene for their use in different structures. Using only three building blocks, four different antigens 1-4 were prepared. The optimized yields after AGA ranged from $36 \%$ to $8 \%$, the lower values corresponding to the longer structures. Exploring BBs with different protecting groups or leaving groups (e.g. phosphate donors) can lead to higher yields at this stage. The larger structures also showed lower deprotection yields, which reinforces the need for more efficient hydrogenation methods. The combination of enzymatic sialylation with AGA proved to be an expeditious approach to the preparation of complex glycans for vaccine development. Compounds 1-3 and 15 were used in competitive ELISA experiments. Structure $\mathbf{1}$ and its dimer $\mathbf{2}$ showed to inhibit the binding of Sp14 polysaccharide with specific murine serum, providing further evidence for the assignment of the branched tetrasaccharide $\mathbf{1}$ as the minimal epitope. At the same time, $\mathbf{1}$ and $\mathbf{2}$ did not deplete the recognition of GBSIII polysaccharide with a mAb, supporting the need for the Glc-GlcNAc-Gal-motif in for binding. Vaccine development can greatly advance with the increased accessibility to the glycans prepared in this work as the elucidation of minimal epitopes opens the possibility to generate analogues in the pursuit of safer and more efficient active ingredients as well as analogues with easier production routines.

\section{Conflicts of interest}

J. L. and M. S. work at GlycoUniverse GmbH \& Co. KGaA, the company that commercializes the synthesis instrument and building blocks. L. del B. and R. A. are employees of GSK group of companies. R. A. is owner of GKS stocks.

\section{Acknowledgements}

This project has received funding from the European Union's Framework Programme for Research and Innovation Horizon 2020 (2014-2020) under the Marie Sklodowska-Curie Grant Agreement No. 675671. We acknowledge Tanistha Gupta for fruitful discussions and Prof. Peter H. Seeberger for valuable feedback. 


\section{Notes and references}

1 M. Guberman and P. H. Seeberger, J. Am. Chem. Soc., 2019, 141, 5581-5592.

2 P. H. Seeberger, Acc. Chem. Res., 2015, 48, 1450-1463.

3 M. Guberman, M. Bräutigam and P. H. Seeberger, Chem. Sci., 2019, 1-96.

4 H. S. Hahm, M. Hurevich and P. H. Seeberger, Nat. Commun., 2016, 7, 1-8.

5 C. F. Liang, H. S. Hahm and P. H. Seeberger, Methods Mol. Biol., 2014, 1229, 3-10.

6 K. Naresh, F. Schumacher, H. S. Hahm and P. H. Seeberger, Chem. Commun., 2017, 53, 9085-9088.

7 A. A. Joseph, A. Pardo-Vargas and P. H. Seeberger, J. Am. Chem. Soc., 2020, 142, 8561-8564.

8 E. Balsells, L. Guillot, H. Nair and M. H. Kyaw, PLoS One, 2017, 12, 1-20.

9 J. N. Weiser, D. M. Ferreira and J. C. Paton, Nat. Rev. Microbiol., 2019, 16, 355-367.

10 B. Lindberg, J. Lonngren and D. A. Powell, Carbohydr. Res., 1977, 58, 177-186.

11 D. Safari, H. A. T. Dekker, J. A. F. Joosten, D. Michalik, A. C. De Souza, R. Adamo, M. Lahmann, A. Sundgren, S. Oscarson, J. P. Kamerling and H. Snippe, Infect. Immun., 2008, 76, 4615-4623.

12 F. Mawas, J. Niggemann, C. Jones, M. J. Corbel, J. P. Kamerling and J. F. G. Vliegenthart, Infect. Immun., 2002, 70, 5107-5114.

13 P. Kaplonek, N. Khan, K. Reppe, B. Schumann, M. Emmadi, M. P. Lisboa, F.-F. Xu, A. D. J. Calow, S. G. Parameswarappa, M. Witzenrath, C. L. Pereira and P. H. Seeberger, Proc. Natl. Acad. Sci. U. S. A., 2018, 115, 13353-13358.

14 H. J. Jennings, K.-G. Rosell and D. L. Kasper, Can. J. Biochem., 1980, 58, 112-120.

15 A. C. Seale, H. Blencowe, F. Bianchi-Jassir, N. Embleton, Q. Bassat, J. Ordi, C. MenCrossed, C. Cutland, C. Briner, J. A. Berkley, J. E. Lawn, C. J. Baker, L. Bartlett, M. G. Gravett, P. T. Heath, M. Ip, K. Le Doare, C. E. Rubens, S. K. Saha, S. Schrag, A. S. Ter Meulen,
J. Vekemans and S. A. Madhi, Clin. Infect. Dis., 2017, 65, S125-S132.

16 A. Nuccitelli, C. D. Rinaudo and D. Maione, Ther. Adv. Vaccines, 2015, 3, 76-90.

17 A. M. Weisner, A. P. Johnson, T. L. Lamagni, E. Arnold, M. Warner, P. T. Heath and A. Efstratiou, Clin. Infect. Dis., 2004, 38, 1203-1208.

18 M. Alpe and S. Oscarson, Carbohydr. Res., 2002, 337, 17151722.

19 C. L. Pereira, A. Geissner, C. Anish and P. H. Seeberger, Angew. Chem., Int. Ed., 2015, 54, 10016-10019.

20 B. Schumann, H. S. Hahm, S. G. Parameswarappa, K. Reppe, A. Wahlbrink, S. Govindan, P. Kaplonek, L. A. Pirofski, M. Witzenrath, C. Anish, C. L. Pereira and P. H. Seeberger, Sci. Transl. Med., 2017, 9, eaaf5347.

21 A. Sundgren, M. Lahmann and S. Oscarson, J. Carbohydr. Chem., 2005, 24, 379-391.

22 E. V. Sukhova, D. V. Yashunsky, Y. E. Tsvetkov, E. A. Kurbatova and N. E. Nifantiev, Russ. Chem. Bull., 2014, 63, 511-521.

23 C.-C. Yu and S. G. Withers, Adv. Synth. Catal., 2015, 357, 1633-1654.

24 V. Pozsgay, J. R. Brisson, H. J. Jennings, S. Allen and J. C. Paulson, J. Org. Chem., 1991, 56, 3377-3385.

25 V. Pozsgay, J. Gaudino, J. C. Paulson and H. J. Jennings, Bioorg. Med. Chem. Lett., 1991, 1, 391-394.

26 V. Cattaneo, F. Carboni, D. Oldrini, R. De Ricco, N. Donadio, I. M. Y. Ros, F. Berti and R. Adamo, Pure Appl. Chem., 2017, 89, 855-875.

27 F. Carboni, R. Adamo, M. Fabbrini, R. De Ricco, V. Cattaneo, B. Brogioni, D. Veggi, V. Pinto, I. Passalacqua, D. Oldrini, et al., Proc. Natl. Acad. Sci. U. S. A., 2017, 114, 5017-5022.

28 Y. Yu, A. Kononov, M. Delbianco and P. H. Seeberger, Chem.Eur. J., 2018, 24, 6075-6078.

29 H. Yu, H. Chokhawala, R. Karpel, H. Yu, B. Wu, J. Zhang, Y. Zhang, Q. Jia and X. Chen, J. Am. Chem. Soc., 2005, 127, 17618-17619.

30 D. Safari, H. A. T. Dekker, G. T. Rijkers, A. van der Ende, J. P. Kamerling and H. Snippe, Glycoconjugate J., 2011, 28, 557-562. 